\title{
Unknotting Operations of Rotation Type
}

\author{
Yoshiyuki OHYAMA
}

Waseda University

\section{Introduction.}

This paper is concerned with knots and links in $S^{3}$. Nakanishi [5] showed that six replacements appearing in the Conway Third Identity are all unknotting operations and determined the number of the equivalence classes for the equivalence relation generated by each replacement for a $\mu$ component link.

Aida [1] generalized two replacements of them to an $n$-gon move and showed that it is an unknotting operation.

In this paper we generalize the rest of six replacements to moves of polygon type similarly as Aida did, and show that any $\mu$ component link can be deformed into a trivial knot by a finite sequence of each of these moves.

We refer to Burde and Zieschang [2] or Rolfsen [6] for standard definitions and results in knot theory.

\section{Preliminary results and main theorems.}

J. H. Conway [3] introduced the potential function for a link with labels. We consider replacements appearing in the Conway Third Identity. Let $L_{1}, L_{2}, L_{3}$ and $L_{4}$ be four links which differ only in one place as is shown in Fig. 2-1.
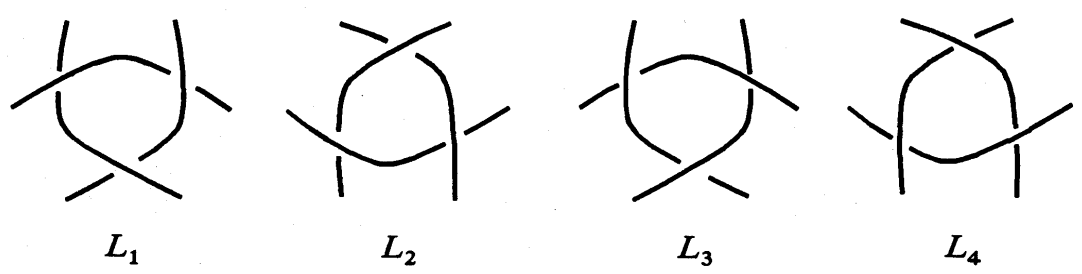

FIGURE 2-1

Nakanishi [5] defined $\Delta_{i j}$-moves as a local move between a link diagram of $L_{i}$ and $L_{j}$, and showed that each $\Delta_{i j}$-move is an unknotting operation. 
Aida [1] generalized a $\Delta_{13}$-move and a $\Delta_{24}$-move to the following $n$-gon moves and proved Theorems 2.2 and 2.3.

DEFINITION 2.1 ([1]). For any integer $n(\geq 3)$, an $n$-gon move is a local move on a link diagram as is indicated in Fig. 2-2.

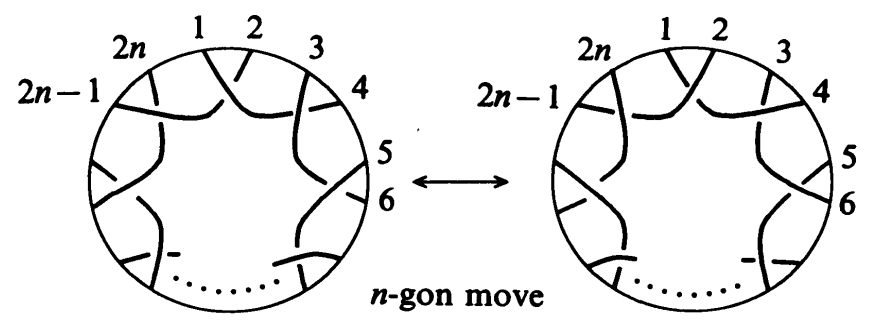

Figure 2-2

THEOREM 2.2 ([1]). For any integer $n(\geq 3)$, every knot can be transformed into a trivial knot by a finite sequence of n-gon moves.

THEOREM 2.3 ([1]). For the equivalence relation generated by $n$-gon moves $(n \geq 3)$, the number of equivalence classes for $\mu$ component links is $2^{\mu-1}$.

Similarly as in Definition 2.1, we generalize $\Delta_{12^{-}}, \Delta_{34}$-moves and $\Delta_{14^{-}}, \Delta_{23^{-}}$-moves to an $n(1)$-move and an $n(2)$-move, respectively.

DEFINITION 2.4. For any integer $n(\geq 4)$, an $n(1)$-move and an $n(2)$-move are local moves depicted in Fig. 2-3 (a) and (b), respectively.

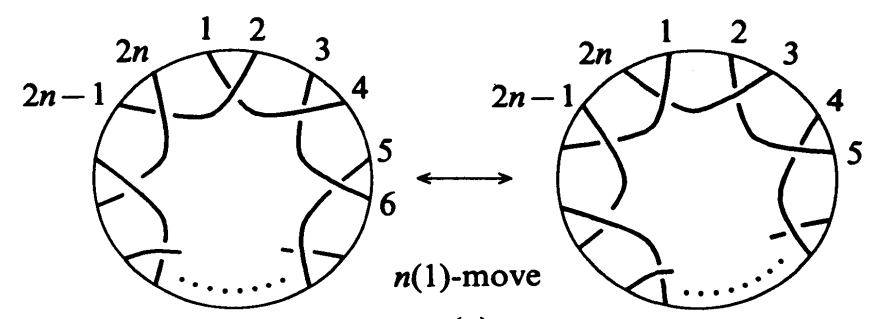

(a)

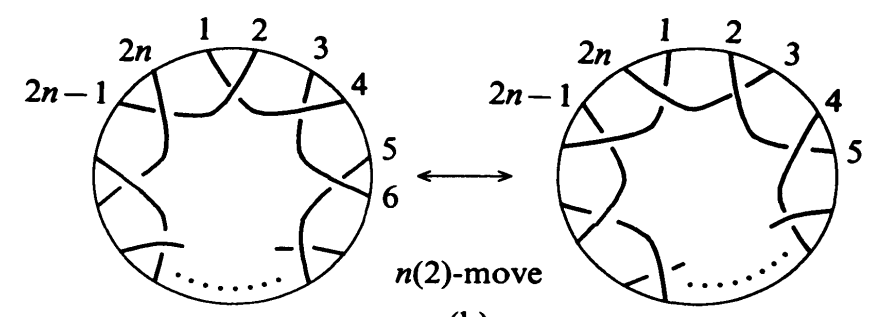

(b)

Figure 2-3 
REMARK. We can consider another move, an $n(1)^{\prime}$-move, in Fig. 2-4. However it is enough to consider an $n(1)$-move since an $n(1)^{\prime}$-move is a mirror image of an $n(1)$ move.

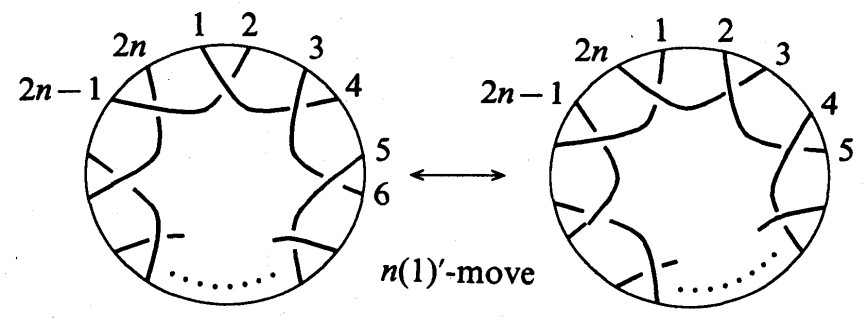

Figure 2-4

As to an $n(1)$-move and an $n(2)$-move, we have the following theorem.

THEOREM 2.5. Any $\mu$ component link can be transformed into a trivial knot by a finite sequence of $n(i)$-moves $(i=1,2)$.

Hoste, Nakanishi and Taniyama [4] proposed a local move on a diagram, which is called an $H(n)$-move. An $H(n)$-move is a local move on a link diagram depicted in Fig. 2-5.

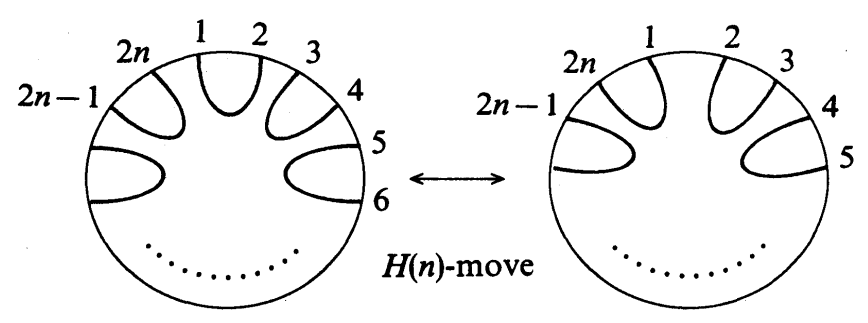

FIGURE 2-5

THEOREM 2.6. ([4]). Given any integer $n(\geq 2)$, any knot can be transformed into a trivial knot by a finite sequence of $H(n)$-moves.

For a knot $K$ and an integer $n, U_{n}(K)$ is defined to be the minimum number of $H(n)$-moves which can transform $K$ into a trivial knot.

THEOREM 2.7 ([4]). For a knot $K$, we have

$$
\lim _{n \rightarrow \infty} U_{n}(K)=1 \text {. }
$$

Similarly as in an $H(n)$-move, $U_{n(i)}(K)$ is defined to be the minimum number of $n(i)$-moves which are necessary to transform a knot $K$ into a trivial knot.

Let $m g(K, p)$ denote the minimum number of generators of the first integral homology group of the $p$-fold cyclic branched covering space of $K$. Then we have Proposition 2.8 . 
Proposition 2.8. For a knot $K$ and an integer $p(\geq 2)$, we have

$$
U_{n(i)}(K) \geq m g(K, p) /(n-1)(p-1) \quad(i=1,2) .
$$

ProOF. By replacing an $H(n)$-move by an $n(i)$-move in the proof of Theorem 4 in [4], we obtain Proposition 2.8.

To prove Theorem 2.7, Hoste, Nakanishi and Taniyama [4] showed that there exists an integer $n$ such that $U_{n}(K)=1$ for a knot $K$. Moreover in the proof of Theorem 2.7 in [4], we can replace a knot $K$ by a $\mu$ component link $L$.

An $n(2)$-move induces an $H(n / 2)$-move if $n$ is even and an $H((n-1) / 2)$-move if $n$ is odd as is shown in Fig. 2-6 (a) and (b), respectively. Therefore we obtain Theorem 2.9.

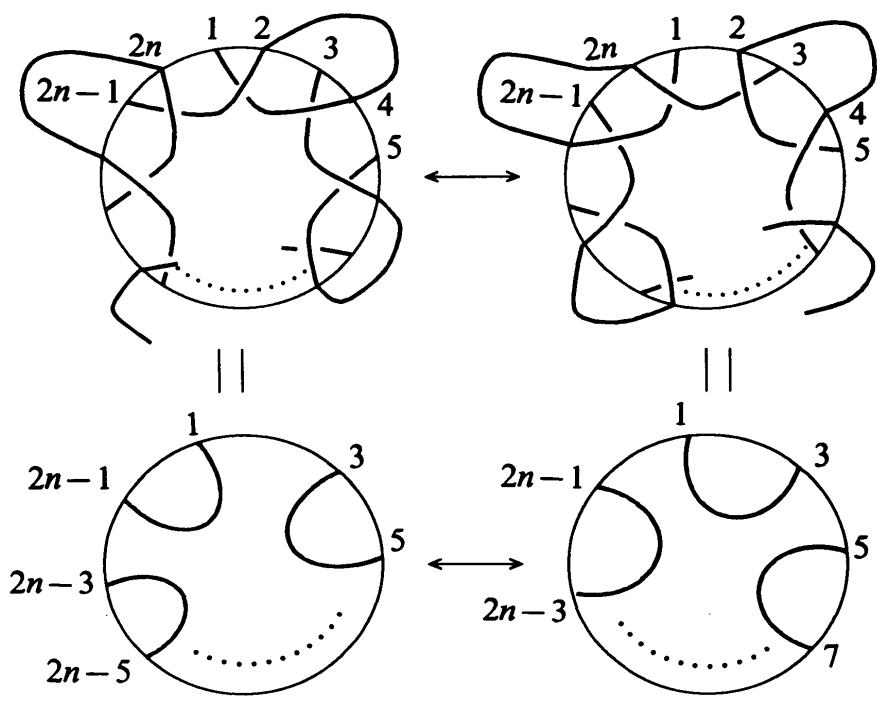

Figure 2-6 (a)

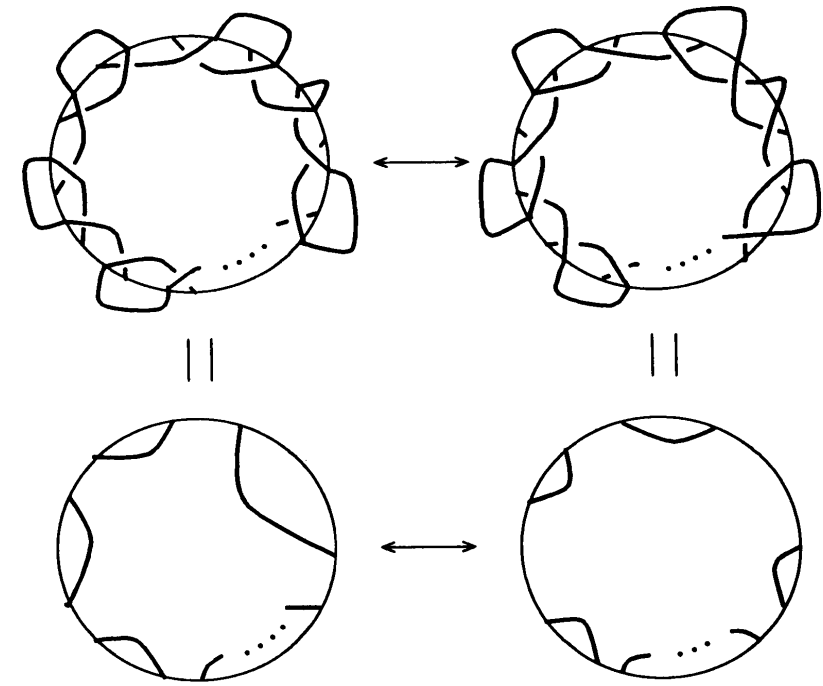

Figure 2-6 (b) 
THEOREM 2.9. For a $\mu$ component link $L$, we have

$$
\lim _{n \rightarrow \infty} U_{n(2)}(L)=1 \text {. }
$$

\section{Proof of Theorem 2.5.}

An $n(2)$-move induces an $H(n / 2)$-move or $H((n-1) / 2)$-move, therefore we have Theorem 2.5 in the case of an $n(2)$-move.

We show Theorem 2.5 in the case of an $n(1)$-move by the following four steps.

Step 1. If we join 1 and 3,2 and $4,2 j-1$ and $2 j(j=3,4, \cdots, n-2)$ at the ends of two tangles of an $n(1)$-move, then an $n(1)$-move induces a move in Fig. 3-1 which can decrease the number of components of a link. Therefore any $\mu$ component link $L$ can be transformed into a knot $K$.

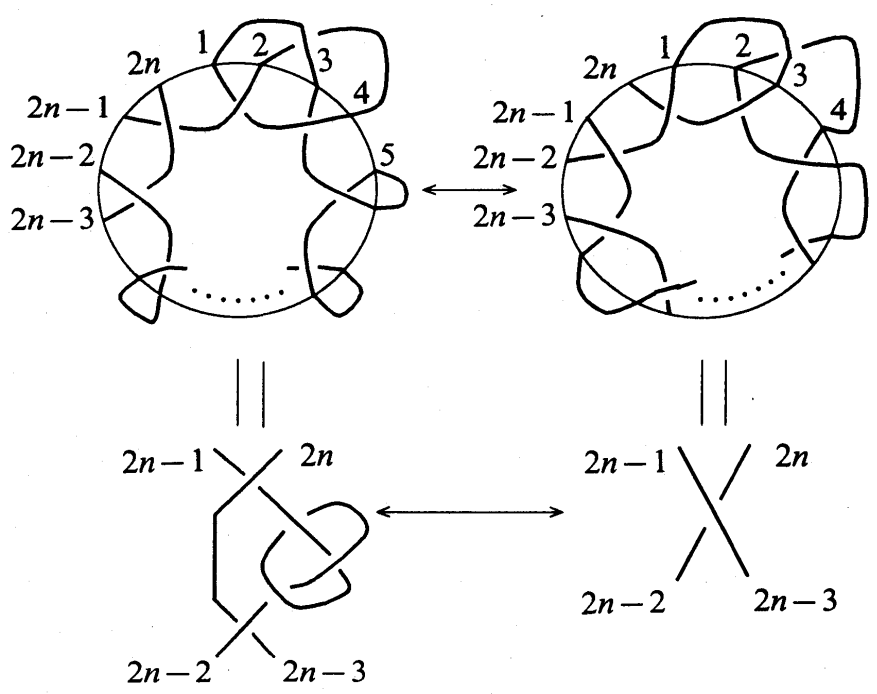

Figure 3-1

Step 2. If we join $2 n$ and $2 n-3$ in Fig. $3-1$, then we have a move in Fig. 3-2 induced from an $n(1)$-move. By this move, any knot $K$ can be transformed into a 2 component link $L=K_{1} \cup K_{2}=K_{1} \#\left(\right.$ a Hopf link), where $K_{1}=K \#($ a trefoil knot).

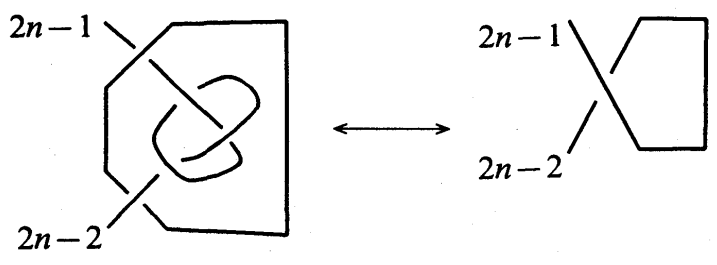

FIGURE 3-2 
Step 3. We show that any two component link $L=K_{1} \#$ (a Hopf link) can be transformed into a link which is a connected sum of a right-handed trefoil and a Hopf link.

First we consider that $n$ is even. In the case $n=4$, we join 3 and 4,1 and 6 at the ends of two tangles of a 4(1)-move. In the case $n \geq 6$, we join $2 j-1$ and $2 j$ $(j=2,3, \cdots, n / 2), 2 k$ and $2 k+1(k=(n+4) / 2,(n+6) / 2, \cdots,(2 n-2) / 2), 1$ and $n+2$ at the ends of two tangles of an $n(1)$-move as is shown in Fig. 3-3. Then an $n(1)$-move induces a move depicted in Fig. 3-4. Since this move induces the move that makes a smoothing at a crossing, it induces the move that changes a crossing. Then by this move we can deform $K_{1}$ into a right-handed trefoil knot.

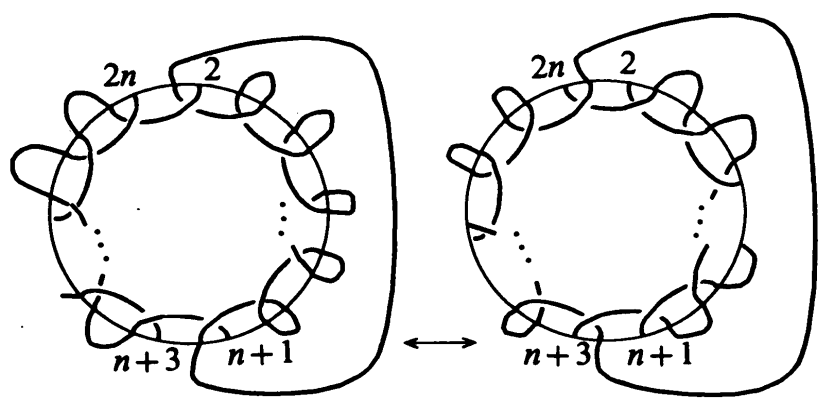

FIGURE 3-3
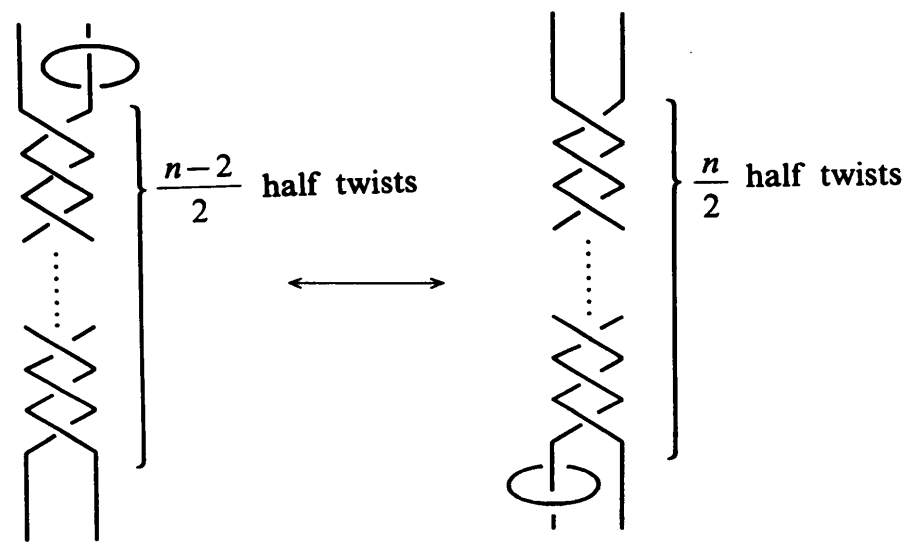

Figure 3-4

In the case that $n$ is odd, we join the ends of two tangles of an $n(1)$-move as the following.

If $n=5$, we join 3 and 4, 5 and 6, 1 and 8 .

If $n \geq 7$, we join $2 j-1$ and $2 j(j=2,3, \cdots,(n+1) / 2), 2 k$ and $2 k+1(k=(n+5) / 2$, $(n+7) / 2, \cdots,(2 n-2) / 2), 1$ and $n+3$. Then an $n(1)$-move induces a move depicted in Fig. 3-5 which induces the move that changes a crossing. Therefore $K_{1}$ can be deformed into a right-handed trefoil knot. 


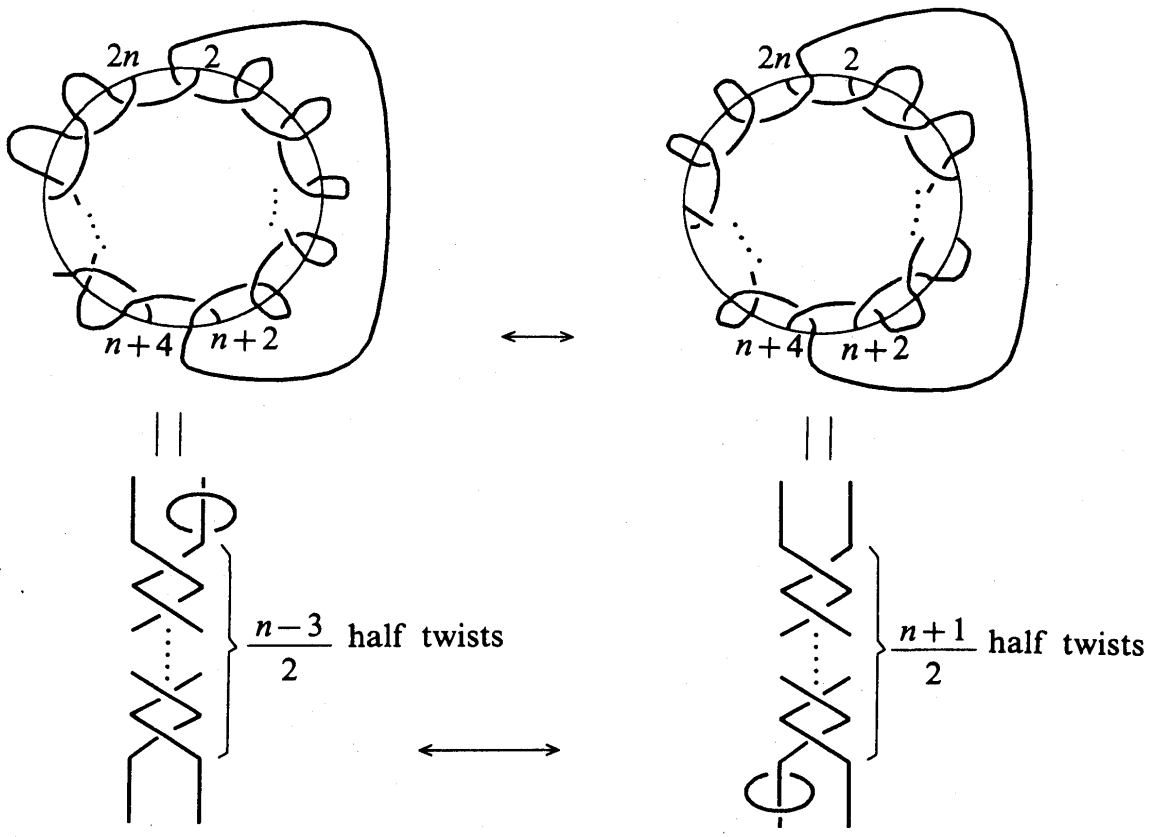

FIGURE 3-5

Now we can transform any link into a link which is a connected sum of a right-handed trefoil and a Hopf link.

Step 4. If we join $2 n$ and $2 n-3,2 n-1$ and $2 n-2$ at the ends of two tangles in Fig. 3-1, then we can deform a link which is a connected sum of a right-handed trefoil and a Hopf link into a trivial knot.

This completes the proof of Theorem 2.5.

\section{References}

[ 1] H. AIDA, Unknotting operations of polygonal type, Tokyo J. Math., 15 (1992), 111-121.

[2] G. Burde and H. Zieschang, Knots, de Cruyter Studies in Math., 5 (1985).

[ 3 ] J. H. Conway, An enumeration of knots and links, and some of their algebraic properties, Computation Problems in Abstract Algebra, 329-358, Pergamon Press, 1969.

[4] J. Hoste, Y. NAKANiSH and K. TANIYAMA, Unknotting operations involving trivial tangles, Osaka J. Math., 27 (1990), 555-566.

[ 5 ] Y. NaKanishi, Replacements in the Conway Third Identity, Tokyo J. Math., 14 (1991), 197-203.

[6] D. Rolfsen, Knots and Links, Math. Lecture Ser., 7 (1976), Publish or Perish.

Present Address:

Department of Mathematics, School of Science and ENGineERING, Waseda UNiversity OHKUBO, SHINJUKU-KU, TOKYO 169-50, JAPAN 